\title{
VARIABILITY OF NGC 3783: FIRST RESULTS FROM AN INTENSIVE GROUND-BASED MONITORING CAMPAIGN
}

\author{
GIOVANNA M. STIRPE \\ Osservatorio Astronomico di Bologna, Via Zamboni 39, 40126 Bologna, Italy
}

The International AGN Watch collaboration undertook an intensive monitoring campaign of the bright Seyfert 1 galaxy NGC 3783 between December 1991 and August 1992, in order to study the variations of the continuum and broad emission lines. Spectroscopic and photometric observations took place at several groundbased observatories and formed the optical/IR counterpart to the UV observations conducted with the IUE (Reichert et al. 1993).

The data allowed to derive the light curves of the optical continuum and of the $\mathrm{H} \beta$ flux with a median sampling of about 2 days. Both curves strongly resemble the UV continuum light curves, and are characterized by a series of rapid, low-amplitude minima, which have counterparts in the UV curves. The optical continuum varied by up to $50 \%$ and the $\mathrm{H} \beta$ flux by up to $30 \%$.

Cross correlation analysis shows that there is no significant delay between the optical and UV continuum curves, which confirms the trend observed between different UV continuum bands. The light curve of $\mathrm{H} \beta$ presents a lag of 8 days with respect to the UV continuum. This lag is longer that those of the high ionization lines (Reichert et al. 1993), indicating that the broad line region (BLR) is stratified. All line vs. continuum lags are shorter than the corresponding ones in NGC 5548, a brighter Seyfert 1 monitored by the collaboration: this indicates that the BLR of NGC 3783 is smaller than that of NGC 5548.

The IR continuum light curve, while displaying some variability ( $20 \%$ in the $\mathrm{K}$ band), does not resemble the UV and optical curves. It is possible that the lower sampling rate and the lower amplitude of the IR variations smoothed the IR light curve, thus hiding any counterparts to the features observed in the other bands.

A detailed description of the results obtained during the ground-based campaign is presented in Stirpe et al. (1993).

\section{Acknowledgements}

This campaign is the result of the combined efforts of many colleagues, who generously contributed to it with their data and time.

\section{References}

Reichert, G.A., et al. 1993, ApJ, in press

Stirpe, G.M., et al. 1993, ApJ, submitted

407

T. J.-L. Courvoisier and A. Blecha: Multi-Wavelength Continuum Emission of AGN, 407.

(C) 1994 IAU. Printed in the Netherlands. 